\title{
Heterogeneous relaxation in supercooled liquids: A density functional theory analysis
}

\author{
Rajesh K. Murarka and Biman Bagchi ${ }^{\mathrm{a})}$ \\ Solid State and Structural Chemistry Unit, Indian Institute of Science, Bangalore-12, India
}

\begin{abstract}
Recent time domain experiments which allow selective study of the relaxation of slower subpopulations among the distributions of local, inhomogeneous regions, have shown the existence of a length scale $(\sim 2-3 \mathrm{~nm})$ beyond which the liquid behaves like a homogeneous liquid. Here we use the density functional theory to calculate the probability of creating a soft localized density fluctuation (density droplet). Theoretical calculation shows that the free energy penalty for creating a local inhomogeneity of small size is much less than that for a large size and that a dense supercooled system is unlikely to sustain inhomogeneity of a length, $\ell_{f}$, which is larger than $5 \sigma$, where $\sigma$ is the molecular diameter. We have calculated both the equilibrium and the nonequilibrium (subsequent to photobleaching) orientational correlation functions with the theoretically obtained inhomogeneous distributions. The nonequilibrium distribution relaxes at a slower rate. A simple two state exchange model has been used to mimic the relaxation of the slow regions to equilibrium; the model shows that the diffusional exchange cannot be the mechanism for the extremely slow relaxation process very near to the glass transition temperature. These results have been compared with recent experimental results.
\end{abstract}

\section{INTRODUCTION}

Supercooled liquids are usually obtained by rapidly cooling liquids sufficiently below their melting point. While relaxation of the various response functions in a liquid above the melting point is exponential-like, the most striking feature of many supercooled liquids is the markedly nonexponential nature of this decay. ${ }^{1}$ Although there have been various types of explanation for this anomalous behavior, the most widely accepted one assumes the existence of spatially heterogeneous dynamics in the supercooled state. ${ }^{2-7} \mathrm{Re}-$ cently photobleaching techniques ${ }^{2,3}$ have been combined with time resolved optical spectroscopy to study the rotational dynamics of the various probe molecules of different sizes in supercooled o-terphenyl (OTP). It has been concluded from these experiments that the structure of the supercooled OTP supports spatially heterogeneous distribution of long-lived, slowly relaxing domains. These studies also indirectly show that at the glass transition temperature $\left(T_{g}\right)$ there exists a length scale $(\sim 2.5 \mathrm{~nm})$ beyond which the liquid is homogeneous. ${ }^{8}$ Recently, Tracht et al. ${ }^{9}$ developed a model independent four-dimensional solid-state NMR experiment that directly measures the length scale of dynamic heterogeneities and they found a length scale of the same order $(\sim 3 \mathrm{~nm})$ for poly(vinyl acetate) (PVAc) at $T_{g}+10 \mathrm{~K}$. The nature and origin (or even the precise definition) of this inhomogeneity is not clear at this point.

The photobleaching techniques allow one to selectively bleach the "fast" regions and thus allow one to study the

\footnotetext{
a) Author to whom correspondence should be addressed; electronic mail: bbagchi@sscu.iisc.ernet.in. Also at the Jawaharlal Nehru Centre for Advanced Scientific Research, Bangalore.
}

relaxation of the "slow" regions. With time, these slow regions redistribute themselves and again an equilibrium distribution is reached. The time taken by the nonequilibrium distribution created by photobleaching to return to equilibrium can be measured by following the rotational motion of probes located in these regions, given, of course, that the labeled probes do not leave the "domains." Ediger and co-workers ${ }^{2-4}$ have reported that the time to return to equilibrium (which they call an exchange time, $\tau_{\text {ex }}$ ) can become considerably larger than the average orientational relaxation time at equilibrium, as the glass transition is approached from higher temperatures.

In this study, we use the density functional theory (DFT) of classical statistical mechanics ${ }^{10}$ to calculate the free energy cost to create localized inhomogeneous regions, each characterized by a density different from the average density of the liquid and their various sizes. DFT is ideal for studying free energy costs of soft density fluctuations which can lead to heterogeneity. It should be noted here that the density need not be the only choice to describe the origin of these heterogeneities. ${ }^{11}$ We find that indeed there exists a maximum length scale of heterogeneity in the supercooled liquid and this length scale is of the same order as has been observed in other experiments. ${ }^{8,9}$ This length scale has a very simple and appealing explanation within DFT. This length is sufficiently small to be energetically favored by the sharp peak in the static structure factor $(S(k))$, but not large enough to encounter the energy cost due to the very low compressibility of dense liquids. We will discuss this point at length later on. While DFT provides some insight into the possible reason for the nonexistence of heterogeneous regions beyond a size, it does not, at least in the form studied 
here, provide also the reason why regions below certain sizes are not relevant. This might be due to the fact that density fluctuations in small regions are not relevant. Molecules in a small region need not be involved in the cooperative slowing down, ${ }^{12}$ a characteristic feature near the glass transition temperature $\left(T_{g}\right)$.

For calculational simplicity, we assume that these heterogeneous regions are spherical in shape. Although the later assumption lead to the analytical calculations in a more tractable form, it might not represent the actual shape of these regions. In fact, there have been numerous computer simulation studies, ${ }^{13-17}$ as well as experiments on dense colloidal liquids, ${ }^{18}$ which show the highly extended nature of these heterogeneous regions. However, we will see later that instead of a sharp cutoff if one extends this simplified assumption to some extent by assuming the heterogeneous regions of continuous density distribution, one would get more or less similar results. The molecular dynamics simulation study of Glotzer and co-workers ${ }^{13-15}$ shows that particles of similar mobility are spatially correlated and thus confirms the existence of dynamical heterogeneities in a supercooled binary Lennard-Jones mixture. In particular, it has been observed that the structural relaxation takes place through the cooperative motion of relatively few, fast-moving particles and these particles form quasi-one-dimensional, stringlike clusters, whose size increases as the glass transition is approached. Particles of low mobility also form clusters, but they are relatively well-ordered and compact, where cluster sizes appear to be insensitive to the temperature. Recently, the three-dimensional time-resolved confocal microscopy experiments on supercooled colloidal liquids and glasses ${ }^{18}$ confirmed the simulation results.

It has already been mentioned that the precise definition of the local inhomogeneities is not known and the equilibrium local density fluctuations may not necessarily be the only choice to describe these heterogeneities; it could be described as well by the local entropy fluctuations. ${ }^{11,19}$ Another key assumption we have made in this study is that the high density regions are associated with low mobility and the low density regions are associated with high mobility. Equivalently, we assume that the dependence of the average relaxation time on the average density of the liquid can be used to determine the local relaxation time in a small localized region. However, recent simulation studies of a model binary Lennard-Jones liquid, ${ }^{15}$ where the mobility of a particle is defined by the magnitude of its maximum displacement over a suitably chosen time interval, demonstrates that the mobility is related to small equilibrium fluctuations in the local potential energy, and, consequently, in the local composition of the mixture. Furthermore, it has been argued that particles of different mobility can be distinguished in terms of the single particle dynamics where the escape rate of the mobile particles from their local environment is higher than the other particles of the sample. Consequently, the dynamic entropy is one of the measures of particle mobilities in supercooled liquids. ${ }^{20}$ Although the simple correlation assumed between the local relaxation time and local density may not be so straightforward, yet several model studies ${ }^{11,19}$ based on this simple assumption were successful in explaining many experimental results specifically the enhancement of translational diffusion near $T_{g}^{11}$ and the anomalous light scattering in the glass transition region. ${ }^{19}$

The present study has several similarities with the beautiful work of Dasgupta and Valls ${ }^{21}$ who carried out extensive Langevin dynamics simulations of binary glass forming liquids, using a free energy functional given by density functional theory. ${ }^{22}$ As the same free energy functional has been used in this work, the density fluctuations considered have the same weights. Despite these similarities, there are several differences also. We consider localized fluctuations, while Dasgupta and Valls considered extended ones. In addition, these authors did not investigate the size of the domains and did not consider orientational relaxation in these heterogeneous domains.

We have studied orientational relaxation in these slow domains by using standard hydrodynamics. The relaxation of the slow domains becomes slower as the glass transition density is approached from below. As it is very difficult to calculate the relaxation of the slow domains to equilibrium, we have introduced a simple two-state exchange model ${ }^{23,24}$ to mimic the behavior and specifically to study whether the diffusional exchange is the defining mechanism for this relaxation. One should note that our model is very similar to the two-state model considered by Chang and Sillescu ${ }^{25}$ to describe the enhancement of translational diffusion relative to rotational motion near $T_{g}$. They have considered an environmental fluctuation model (EFM) where the environment of each molecule fluctuates between only two states, "slow" and "fast." The corresponding master equations are of the same form as in our model except we have not considered the Fickian diffusion term. Our goal is to investigate whether the very slow relaxation observed near $T_{g}$ can be explained by the simple diffusional exchange mechanism.

It is worth mentioning here that the two-state scenario has often been used as a modeling approach to rationalize various kinds of phenomena. ${ }^{26}$ Particularly, in the context of glass transition, there are several studies based on this scenario. $^{27-30}$ Recently Kieffer et al $^{31}$ used this concept extensively to fit their experimental results where the temperature dependence of the complex mechanical modulus of various glass forming liquids was determined on a nanometer scale throughout the transition range by using Brillouin light scattering. In their approach, which is based on simple Boltzmann statistics, the system is partitioned between two distinct structural states, one with the characteristics of a viscoelastic fluid, and the other one being rigid and can respond only elastically. Of course, in a real situation, a distribution of structural states can coexist at various degrees of supercooling. Furthermore, it was postulated that these localized structural fluctuations are separated by a diffusive interface; as both states are amorphous in nature there are no distinct interfaces between these structural domains and the surface tension terms are negligible. In particular, it was found that there is a gradual structural transition from the glassy to the viscoelastic state at high temperatures. The most remarkable finding of this study is that the temperature dependence of the inherent structure energies of a binary Lennard-Jones liquid, studied by Sastry et l. $^{32}$ using com- 
puter simulations, can be well fit by this two-state model and thus provides support for the concept that thermodynamically driven structural transitions underlie the glass transition phenomena.

It is interesting to note that the free energy functional form used in this study has also recently been used to investigate the stability of a structure in the metastable state which is in between the homogeneous liquid state and the regular crystalline state. ${ }^{33}$ In that case, the heterogeneous density distribution was expressed by the superposition of Gaussian profiles centered on an amorphous lattice and was characterized by a variational parameter $\alpha$, which represented the degree of localization of the particles in the system. A free energy minimum corresponding to a metastable supercooled state of less localized structure was found, in addition to the highly localized "hard sphere glass." 34 Most interestingly, it was found that this minimum does not occur if the underlying lattice is taken as a regular crystalline one. Although this study supports the view of the existence of a heterogeneous density distribution, the absence of a minimum in the crystalline configuration is surprising, but it might be a pathology of hard sphere system.

The organization of the rest of the paper is as follows. Section II provides the expressions necessary to calculate the probability distribution of inhomogeneous regions using density functional theory (DFT). The numerical results of the probability distribution are presented in Sec. III. Section IV describes the rotational dynamics of the molecules in relaxing inhomogeneous domains. The relaxation of nonequilibrium density distribution is described in terms of two-state exchange model in Sec. V. Finally, concluding remarks are presented in Sec. VI.

\section{PROBABILITY DISTRIBUTION OF INHOMOGENEOUS REGIONS}

The normalized probability distribution having density fluctuation $(\delta \rho)$ is given by

$$
P(\{\delta \rho\})=\frac{\exp (-\beta \Delta F(\{\delta \rho\}))}{\int d \rho \exp (-\beta \Delta F(\{\delta \rho\}))},
$$

where $\Delta F=F[\rho]-F[\bar{\rho}]$ is the excess free energy required for creating an heterogeneous region of density $\rho$ in the uniform liquid of density $\bar{\rho} . \beta$ is the inverse of the Boltzmann constant $\left(k_{B}\right)$ times the absolute temperature $(T)$.

The heterogeneous regions are spherical in shape as per our assumption and the density fluctuation can be given approximately by the following Gaussian form:

$$
\delta \rho(\mathbf{r})=\delta \rho(\mathbf{r}=\mathbf{0}) \exp \left(-\frac{(r-\bar{r})^{2}}{R_{I}^{2}}\right)
$$

Here $\bar{r}$ represents the mean position of the fluctuation and for simplicity it is assumed to be taken as the origin $(\mathbf{r}=\mathbf{0})$. $\delta \rho(\mathbf{r}=\mathbf{0})$ gives the maximum value of the fluctuation. $R_{I}$ determines the spread of this fluctuation from the origin.

We have also chosen an alternative form of the density fluctuation to check the sensitivity of the results of the as- sumption of continuous density distribution. The alternative distribution is given by the following step function expression:

$$
\delta \rho(\mathbf{r})=\delta \rho(\mathbf{r}=\mathbf{0}) H\left(R_{I}-|\mathbf{r}|\right),
$$

where similarly the maximum value of the fluctuation is given by $\delta \rho(\mathbf{r}=\mathbf{0}) . H\left(R_{I}-|\mathbf{r}|\right)$ is the heaviside step function and takes the following simple form:

$$
H\left(R_{I}-|\mathbf{r}|\right)=\begin{array}{ll}
1, & R_{I}>|\mathbf{r}| \\
0, & R_{I}<|\mathbf{r}| .
\end{array}
$$

Here $R_{I}$ is the radius of the spherical heterogeneous region.

The form of the density functional $F[\rho]$ is approximately given by the following functional Taylor series expansion against $\rho(\mathbf{r})$ truncated at the second order term: ${ }^{22,35}$

$$
\begin{aligned}
\beta F[\rho]= & \beta F[\bar{\rho}]+\int d \mathbf{r}[\rho(\mathbf{r}) \ln (\rho(\mathbf{r}) / \bar{\rho})-\delta \rho(\mathbf{r})] \\
& -\frac{1}{2} \iint d \mathbf{r} d \mathbf{r}^{\prime} c^{(2)}\left(\left|\mathbf{r}-\mathbf{r}^{\prime}\right|\right) \delta \rho(\mathbf{r}) \delta \rho\left(\mathbf{r}^{\prime}\right),
\end{aligned}
$$

where $F[\bar{\rho}]$ is the free energy of the liquid at the uniform density $\bar{\rho}$, and $\delta \rho(\mathbf{r})=\rho(\mathbf{r})-\bar{\rho}$ measures the deviation of the density from $\bar{\rho}$ at the spatial point $\mathbf{r}$. $\beta$ is the inverse of the Boltzmann constant $\left(k_{B}\right)$ times the absolute temperature $(T) . c^{(2)}(r)$ is the direct pair correlation function of the uniform liquid of density $\bar{\rho}$. To calculate the direct pair correlation function, $c^{(2)}(r)$, we have used the well-known Percus-Yevick approximation ${ }^{36}$ which is appropriate for the hard sphere liquid.

\section{NUMERICAL CALCULATION OF THE DISTRIBUTION, $\boldsymbol{P}(\boldsymbol{\rho})$}

We have calculated the corresponding free energy cost to create density fluctuations of various size. The calculation has been carried out for both types of density fluctuation form described in Sec. II. The scaled density of the uniform liquid, $\bar{\rho}^{*}=\bar{\rho} \sigma^{3}$ ( $\sigma$ is the hard sphere diameter) is assumed as 1.04. We have also calculated the probability distribution for different sizes of the localized region. This has been done by creating both positive and negative fluctuations in density relative to the uniform liquid. The density $\left(\rho^{*}=\rho \sigma^{3}\right)$ is varied here continuously and the distribution is calculated by using Eq. (1).

In Fig. 1, we plot the distribution $P(\{\delta \rho\})$ as a function of $\delta \rho$, for fluctuations of three different sizes, $R_{I}=4.0 \sigma$, $R_{I}=2.5 \sigma$ and $R_{I}=1.5 \sigma$, respectively, where $\sigma$ is again the hard sphere diameter. The calculation has been performed using the Gaussian form of the density fluctuation (Eq. (2)). It can be seen from this figure that the distribution is nearly Gaussian. This is because the free energy surface is nearly harmonic-interestingly the anharmonicity increases with decrease in size. The free energy required to create a heterogeneous region of small size is much less compared to that for a large size. This large free energy cost to create larger inhomogeneous regions follows directly from the following approximate expression for the probability of fluctuation of the wave number dependent density, $\rho_{\mathbf{k}}$ : 


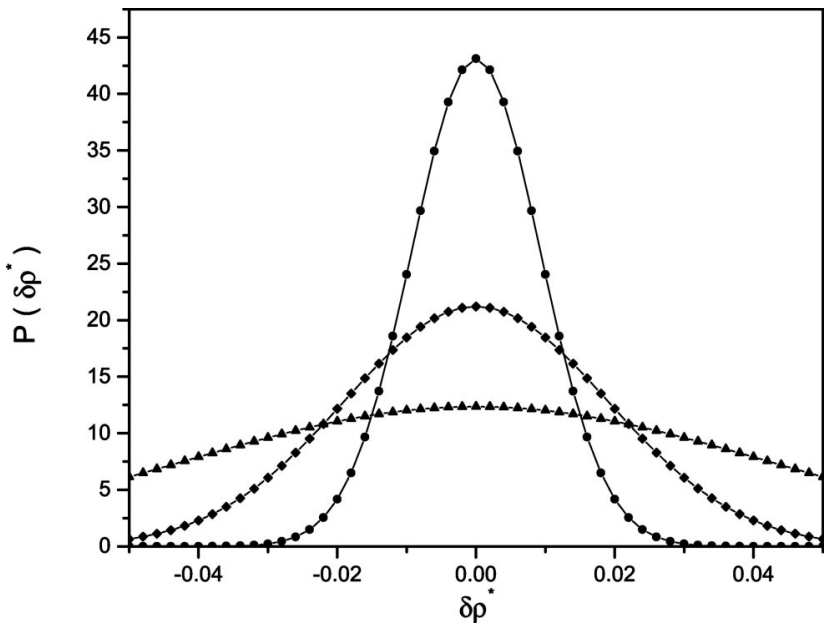

FIG. 1. The normalized probability distribution, $P(\{\delta \rho\})$, plotted as a function of local density fluctuation, $\delta \rho$, for three different sizes $\left(R_{I}\right)$ of the local inhomogeneities. Note that the reduced density of the uniform liquid, $\bar{\rho}^{*}\left(=\bar{\rho} \sigma^{3}\right)$ is assumed to be 1.04 . The solid triangles denote the calculated results for $R_{I}=1.5 \sigma$, solid diamonds for $R_{I}=2.5 \sigma$, and the solid circles for $R_{I}=4.0 \sigma$. The solid lines are simply an aid to the human eye. For further discussion, see the text.

$$
P\left(\left\{\rho_{\mathbf{k}}\right\}\right)=\frac{1}{\sqrt{\pi S(\mathbf{k})}} \exp \left(-\frac{\rho_{\mathbf{k}} \rho_{-\mathbf{k}}}{S(\mathbf{k})}\right),
$$

where $\rho_{\mathbf{k}}$ is the spatial Fourier transform of $\rho(\mathbf{r})$,

$$
\rho_{\mathbf{k}}=\int d \mathbf{r} \exp (-i \mathbf{k} . \mathbf{r}) \rho(\mathbf{r}),
$$

and $S(\mathbf{k})$ is the static structure factor of the supercooled liquid. In a supercooled liquid $S(\mathbf{k})$ is nearly zero for small wave numbers (because of very low compressibility), hence density fluctuation can take place only in intermediate wave numbers where $S(\mathbf{k})$ is large.

As can be seen from Fig. 1, fluctuations of small size have a broader distribution and the width of the distribution becomes narrower as size increases. The probability of having fluctuations of small size is finite even when the fluctuations in density are very high whereas it is almost zero for the large size. For the size, $R_{I}=4.0 \sigma$, the spread of the distribution of the localized inhomogeneous regions becomes rather small. This clearly demonstrates that it is easier to create the soft fluctuations and after certain length scales $\left(\ell_{f} \geqslant 5 \sigma\right)$ the system behaves like a homogeneous liquid.

It should be noted here that the distributions calculated using the step function form of the density fluctuation [Eqs. (3) and (4)] also show similar types of behavior.

To compare the results obtained for hard spheres (Fig. 1) with the systems interacting via continuous potentials, the aforementioned calculation for the probability distribution has also been carried out for the Lennard-Jones (LJ) system. The direct pair correlation function, $c^{(2)}(r)$, for this system is calculated by solving the Ornstein-Zernike equation for neat supercooled liquid by using the soft mean spherical approximation (SMSA) ${ }^{37}$ SMSA is known to provide a reasonably accurate description of static correlation functions in dense liquids. The calculation is performed at a reduced temperature $T^{*}\left(=k_{B} T / \epsilon\right)$ equal to unity and the reduced den-

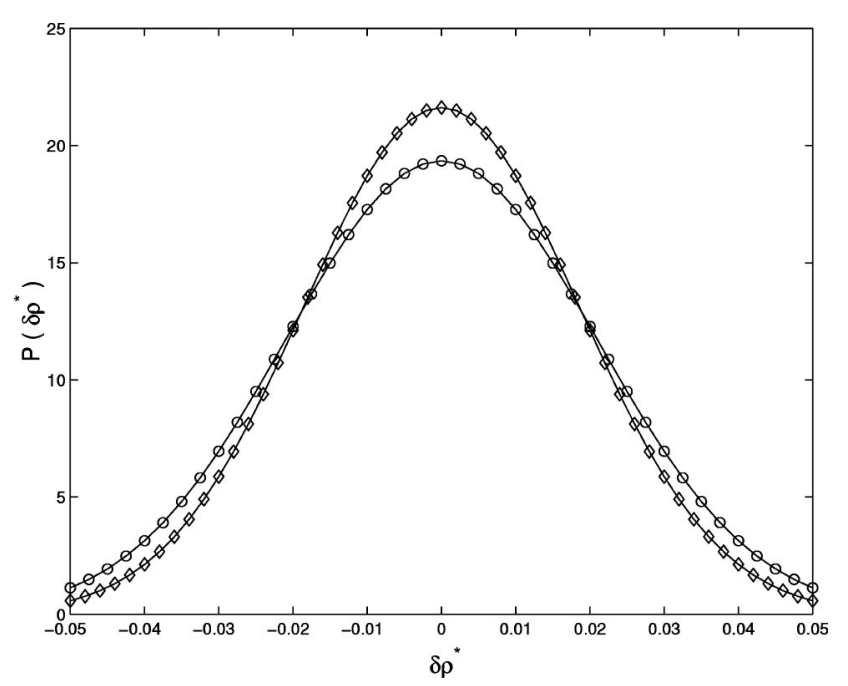

FIG. 2. The same quantity depicted in Fig. 1 but now the results are compared with those calculated for continuous potentials for a particular size of the heterogeneity. For the continuous potential, the calculation is done at a reduced temperature $T^{*}\left(=k_{B} T / \epsilon\right)$ equal to unity and the reduced density of the uniform liquid, $\bar{\rho}^{*}=\bar{\rho} \sigma_{\mathrm{LJ}}^{3}$ is set to 1.0142 . The calculation is performed for the heterogeneity of size $R_{I}=2.5 \sigma_{\mathrm{LJ}}$, where $\sigma_{\mathrm{LJ}}$ is the LJ diameter. This corresponds approximately to the size of $2.479 \sigma$, where $\sigma$ is the effective hard sphere diameter. Open diamonds are the results for the hard sphere system and open circles for the continuous potentials. Solid lines are provided as a guide to the eye. For further details, see the text.

sity of the uniform liquid, $\bar{\rho}^{*}=\bar{\rho} \sigma_{\mathrm{LJ}}^{3}$ (where $\sigma_{\mathrm{LJ}}$ is the LJ diameter) is set at 1.0142 . This system can be mapped into the hard sphere fluid characterized by a density and temperature dependent effective diameter, ${ }^{38}$ by employing the wellknown Weeks-Chandler-Anderson (WCA) perturbation scheme. ${ }^{39}$ In Fig. 2, a comparison is made for a particular size of the localized region, $R_{I}=2.5 \sigma_{\mathrm{LJ}}$, which corresponds to approximately the size of $2.479 \sigma$ ( $\sigma$ is the hard sphere diameter). It shows that the size of the localized density fluctuations will be somewhat larger compared to the hard sphere result. In the case of continuous potentials one has an attractive part and as a consequence there is an increase in spatial correlation length. In addition, the system has now become more compressible than the hard sphere liquid and thus allows for the persistence of localized fluctuations of larger wavelength.

The results presented above do not consider the surface tension term which can arise because regions of high density are likely to be surrounded by regions of low density. The localized regions which exist in the highly supercooled liquid can be represented by the "density droplets" much as in the same spirit of the "entropy droplet" picture of Wolynes. ${ }^{40,41}$ To create these density droplets one would have extra surface energy term in addition to the energy cost given by the density functional expression [Eq. (5)]. Thus the total free energy of the droplet can be expressed as a function of the radius of the droplet $\left(R_{I}\right)$,

$$
\Delta F_{T}\left(R_{I}\right)=\Delta F(\{\delta \rho\})+4 \pi R_{I}^{2} \gamma,
$$

where $\Delta F(\{\delta \rho\})$ is the excess free energy required for creating the droplet of density $\rho$ in the uniform liquid of density $\bar{\rho}$ and $\gamma$ is the surface free energy per unit area. Note that the 


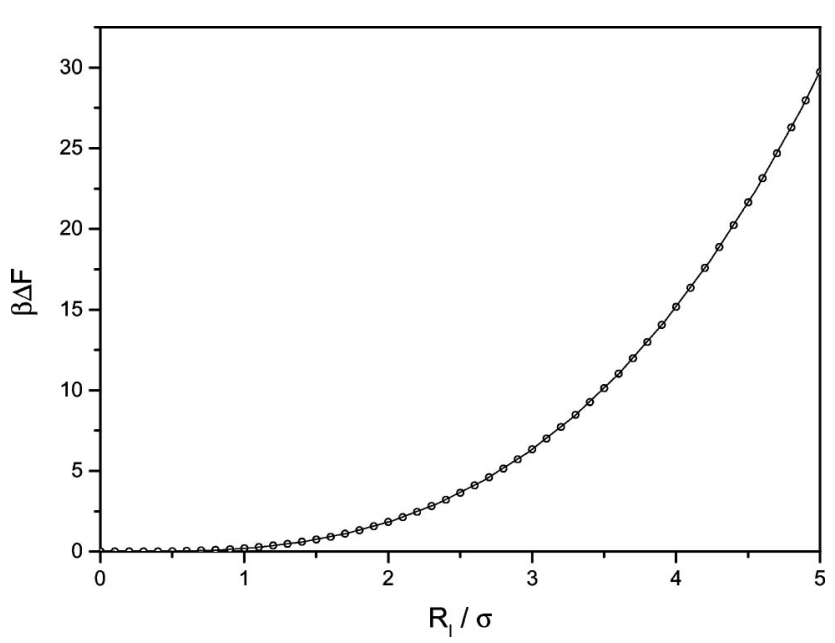

FIG. 3. The relative excess free energy, $\beta \Delta F$, as a function of the droplet radius $\left(R_{I}\right)$ for a fluctuations of fixed amplitude, $\delta \rho^{*}=0.05$. This excess free energy has been calculated using Eq. (5), where the Percus-Yevick approximations (PY) is used for the direct pair correlation function. Open circles are the calculated values and the solid line is the cubic polynomial fit in $R_{I}$. The fit parameters are as follows: $\beta \Delta F=0.01094-0.06293 R_{I}$ $+0.0071 R_{I}^{2}+0.23871 R_{I}^{3}$. Note that the free energy is scaled by $k_{B} T$ $(=1 / \beta)$ and $R_{I}$ is scaled by $\sigma$ ( $\sigma$ is the hard sphere diameter). For detailed discussion, see the text.

contributions from both of the two terms in the right hand side of the above expression are positive. This is in contrast to the conventional classical nucleation theory, where the formation of a new thermodynamically more stable phase in the original mother phase is preferred, but the cost due to surface energy acts against the growth of the embryo of the new phase. As a result of these two competing effects, there exists a free energy barrier corresponding to a nucleus of a critical size. Variation of the excess free energy of the droplet of particular density calculated using the DFT expression [Eq. (5)] as a function of the droplet radius $\left(R_{I}\right)$ is shown in Fig. 3. As can be seen from this figure, this energy grows with the radius of the droplet. In order to see the functional form of this growth, we have fitted the data to polynomials in $R_{I}$. The details of the fit parameters are given in the caption of Fig. 3. While the surface energy term grows as $R_{I}^{2}$, the cost of the density fluctuation grows as $R_{I}^{3}$. Thus the main effect of the inclusion of the surface energy term would be to reduce the probability of small size droplets rather than the droplets of large size and the small scale fluctuations will become less probable. However, as the amplitude of these fluctuations is small and the symmetry of the liquid is almost preserved, the surface free energy per unit area $(\gamma)$ is expected to be very small. Consequently, the surface energy contribution should have a less pronounced effect unlike in the case of thermodynamically driven first order transitions.

\section{ROTATIONAL DYNAMICS IN RELAXING INHOMOGENEOUS DOMAINS}

As discussed in Sec. I, the nonexponential decay of the orientational correlation functions can be described, at least partly, considering spatial heterogeneous dynamics of the supercooled liquid and this has been confirmed recently by the photobleaching experiments. ${ }^{2,3} \mathrm{We}$ assume that the local inhomogeneities having different relaxation times are described by different local densities.

The density dependence of the relaxation times for supercooled liquids is often described by the well-known Vogel-Tammann-Fulcher (VTF) equation, ${ }^{42}$

$$
\tau(\rho)=\tau_{0} \exp \left(\frac{C}{\rho_{g}-\rho}\right),
$$

where the constant $C$ is proportional to the activation energy for the relaxation rate. $\rho_{g}$ is the density at the glass transition point.

The orientational correlation function averaged over the probability distribution of the local relaxation times, $P(\tau)$, can be expressed as

$$
\begin{aligned}
\left\langle C_{2 R}(t)\right\rangle & =\int d \tau P(\tau) \exp \left(-\frac{t}{\tau_{2 R}(\tau)}\right) \\
& \equiv \int d \rho P(\rho) \exp \left(-\frac{t}{\tau_{2 R}(\rho)}\right),
\end{aligned}
$$

where $\tau_{2 R}(\rho)$ defines the rotational relaxation times in the domains having different local densities. It is assumed to be given by the following expression: ${ }^{36}$

$$
\tau_{2 R}(\rho)=\frac{1}{6 D_{R}(\rho)}=\frac{8 \pi \eta(\rho) R^{3}}{6 k_{B} T} .
$$

In the above expression, $D_{R}$ is the local rotational diffusion coefficient and the last equality results by using the wellknown Debye relation, $D_{R}=k_{B} T / 8 \pi \eta R^{3}$, where $R$ is the molecular radius. The density dependence of the viscosity, $\eta(\rho)$, is assumed to described by the VTF equation.

The average rotational correlation time is calculated as usual by integrating the orientational correlation function as

$$
\left\langle\tau_{2 R}\right\rangle=\int_{0}^{\infty} d t\left\langle C_{2 R}(t)\right\rangle .
$$

It was observed in recent experiments of Ediger $e t a l .^{2,3}$ that the average rotational correlation time increases with the fraction of probe molecules that have been photobleached. This led them to conclude that there exists a spatially heterogeneous distribution of local relaxation times and the increase in the correlation time is due to the selective destruction of subset of probe molecules in more mobile environments. To study the effect of this selective photobleaching on the rotational dynamics of the molecules we have created a nonequilibrium probability distribution of density by tagging only the slow regions relative to the uniform liquid. This is shown by the hatched area in Fig. 4. We have used the respective normalized distributions thus obtained to calculate the equilibrium and nonequilibrium orientational correlation function $\left\langle C_{2 R}(t)\right\rangle$. Figure 5 displays the decay behavior of these correlation functions for a particular size of the local inhomogeneity, $R_{I}=2.5 \sigma$, at the average density of the liquid, $\bar{\rho}^{*}=1.04$ (glass transition density $\rho_{g}^{*}$ is taken as 1.1). The decay is nonexponential in nature and the value of the average correlation time has increased almost by a factor of 1.8 in the nonequilibrium ensemble. Figure 6 


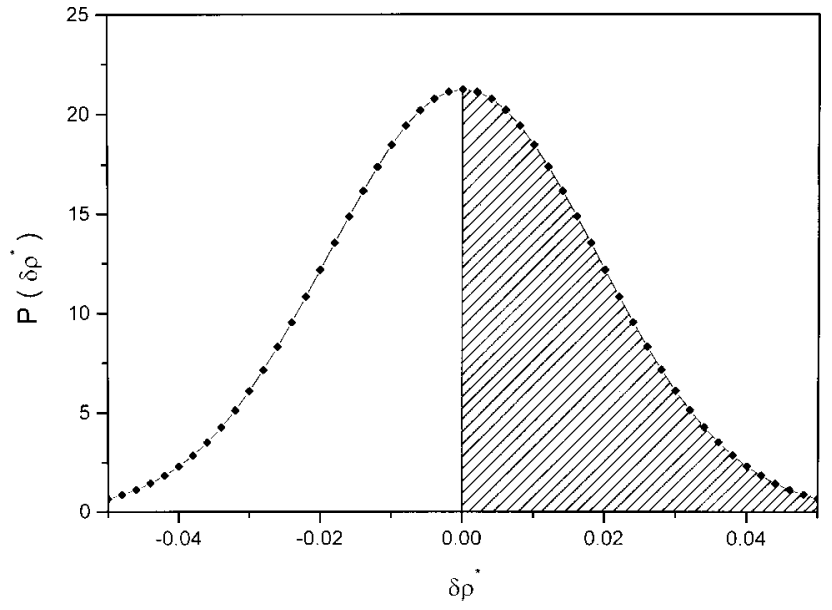

FIG. 4. Schematic representation of the unnormalized nonequilibrium distribution of density for a particular size of the inhomogeneity, $R_{I}=2.5 \sigma$. The tagging of only slow regions relative to the uniform liquid in the normalized equilibrium distribution is shown by the hatched area.

clearly shows that this difference in average correlation time will increase as we continue to approach the glass transition density. The physical origin of this increase is easy to understand. With the increase in density, although the faster regions become increasingly slow, the slower regions become slower at a faster rate due to the divergence in relaxation time near the glass transition point. Thus the structure of the supercooled liquid becomes dynamically more heterogeneous as the density increases toward the glass transition density.

Even in the idealistic model described above, it is nontrivial to formulate the relaxation of the nonequilibrium distribution of density to the equilibrium one. In order to have a qualitative idea about how this relaxation can explain the experimental results, we have carried out an approximate

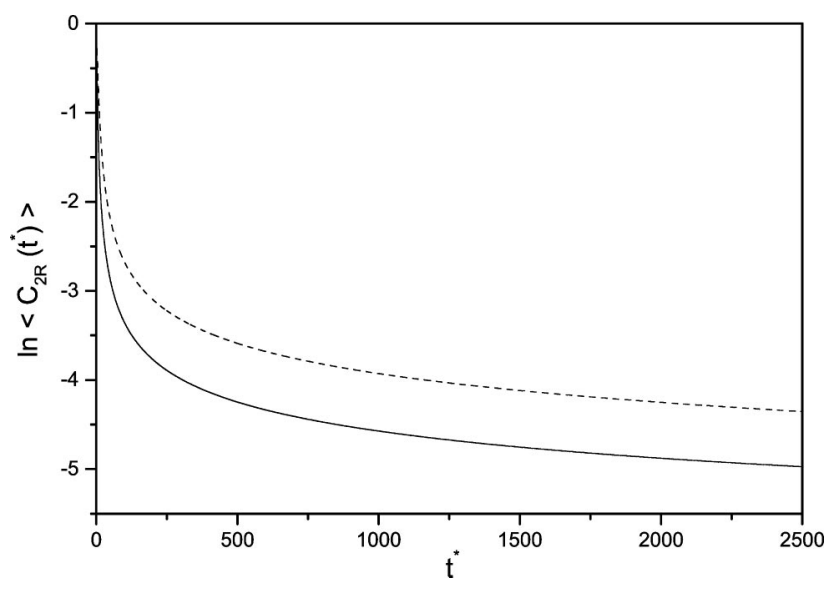

FIG. 5. The decay behavior of both the equilibrium and nonequilibrium orientational correlation functions, $\left\langle C_{2 R}(t)\right\rangle$, where time $t$ is plotted for $R_{I}$ $=2.5 \sigma$. The average density of the liquid $\left(\bar{\rho}^{*}\right)$ and the density at the glass transition point $\left(\rho_{g}^{*}\right)$ are 1.04 and 1.1 , respectively. The solid line indicates the decay at equilibrium and the dashed line immediately after creating the nonequilibrium distribution. In the nonequilibrium ensemble the average rotational correlation time has increased and is almost 1.8 times the equilibrium value. Note that the time $t$ is scaled by $\left[m \sigma^{2} / k_{B} T\right]^{1 / 2}$. For further discussion, see the text.

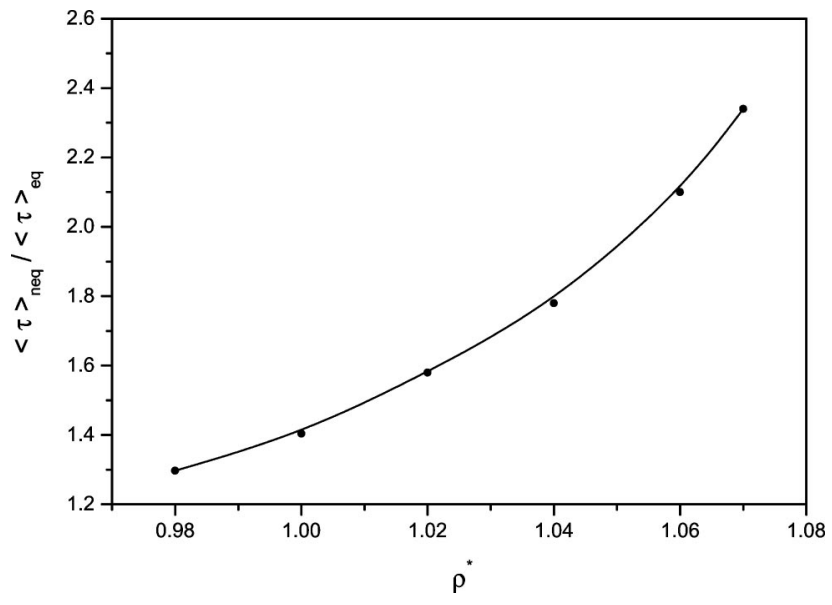

FIG. 6. The average ratio of the rotational correlation times in the nonequilibrium ensemble $\left(\langle\tau\rangle_{\text {neq }}\right)$ to the equilibrium values $\left(\langle\tau\rangle_{\text {eq }}\right)$ as a function of the scaled average density $\bar{\rho}^{*}\left(=\bar{\rho} \sigma^{3}\right)$ for the regions of size, $R_{I}=2.5 \sigma$. The glass transition density $\left(\rho_{g}^{*}\right)$ is assumed to be 1.1. The solid line is simply an aid to the human eye. Note that the correlation times are scaled by $\left[m \sigma^{2} / k_{B} T\right]^{1 / 2}$. For further details, see the text.

calculation based on a simplified noninteracting, two-state exchange model, described in the following section.

\section{TWO-STATE EXCHANGE MODEL FOR RELAXATION OF NONEQUILIBRIUM DENSITY DISTRIBUTION}

We consider the system consists of only two dynamical states where the dynamics in one of these states is fast and slow in the other. These two states are characterized by the two different densities, $\rho_{1}^{*}$ and $\rho_{2}^{*}$, respectively and by two local rotation times, $\tau_{1}$ and $\tau_{2}$.

Assuming there is an exchange between these two different dynamical regions at a rate, $k_{\mathrm{ex}}$, the time dependent probability distribution for state 1 within this model is given by the following expression:

$p_{1}(t)=\frac{1}{2}\left(1+p(t=0) e^{-2 k_{\mathrm{ex}} t}\right)$,

and similarly the same for state 2 is given by

$$
p_{2}(t)=\frac{1}{2}\left(1-p(t=0) e^{-2 k_{\mathrm{ex}} t}\right),
$$

where $p(t=0)=p_{1}(t=0)-p_{2}(t=0)$.

Now the orientational correlation function averaged over different time intervals $\left(t_{d}\right)$ simply can be expressed as

$$
\begin{aligned}
\left\langle C_{2}(t)\right\rangle\left(t_{d}\right)= & \Sigma_{i=1}^{2} p_{i}\left(t_{d}\right) \exp \left(-\frac{t}{\tau_{i}}\right) \\
= & \frac{1}{2}\left(1+\left(p_{1}\left(t_{d}=0\right)-p_{2}\left(t_{d}=0\right)\right) e^{-2 k_{\mathrm{ex}} t_{d}}\right) \\
& \times \exp \left(-\frac{t}{\tau_{1}}\right)+\frac{1}{2}\left(1-\left(p_{1}\left(t_{d}=0\right)\right.\right. \\
& \left.\left.-p_{2}\left(t_{d}=0\right)\right) e^{-2 k_{\mathrm{ex}} t}\right) \exp \left(-\frac{t}{\tau_{2}}\right)
\end{aligned}
$$

Here time interval $t_{d}$ measures the delay time after creating a nonequilibrium distribution which in this model is considered to be represented by slow regions only and thus $p_{1}\left(t_{d}\right.$ 
$=0)=0$ and $p_{2}\left(t_{d}=0\right)=1$. With an increase in time $t_{d}$, the distribution achieves equilibrium when both of the two states have equal probabilities, $p_{1}=p_{2}=\frac{1}{2}$.

The time integration of the correlation function again gives the average rotational correlation time at time $t_{d}$,

$$
\langle\tau\rangle_{\text {neq }}\left(t_{d}\right)=\int_{0}^{\infty} d t\left\langle C_{2}(t)\right\rangle\left(t_{d}\right) .
$$

This nonequilibrium average, $\langle\tau\rangle_{\text {neq }}\left(t_{d}\right)$ is a marker of the relaxation of the distribution. Ediger and co-workers ${ }^{2}$ found that this relaxation occurs at a very slow rate and the exchange time $\left(\tau_{\text {ex }}\right)$ shows strong temperature dependence near the glass transition temperature $\left(T_{g}\right)$. The microscopic mechanism behind this very slow exchange is yet to be understood.

To analyze the origin of this very slow decay, we have followed a procedure already used in experiments. ${ }^{3}$ We start by defining a normalized correlation function, $C_{\tau}\left(t_{d}\right)$, as

$$
C_{\tau}\left(t_{d}\right)=\frac{\left[\langle\tau\rangle_{\mathrm{neq}}\left(t_{d}\right) /\langle\tau\rangle_{\mathrm{eq}}-1\right]}{\left[\langle\tau\rangle_{\text {neq }} /\langle\tau\rangle_{\mathrm{eq}}-1\right]} .
$$

$C_{\tau}\left(t_{d}\right)$ so defined starts at 1 at $t_{d}=0$ and decays to 0 in the limit of $t_{d}=\infty$. Using this normalized correlation function, the exchange time can be defined as

$$
\tau_{\mathrm{ex}}=\int_{0}^{\infty} d t_{d} C_{\tau}\left(t_{d}\right) .
$$

Use of Eqs. (14) and (15) in Eq. (16) gives

$$
\tau_{\mathrm{ex}}=\frac{1}{2 k_{\mathrm{ex}}} .
$$

That is, within the symmetric two-state model, $C_{\tau}\left(t_{d}\right)$ indeed probes the exchange process. However, in the case of a nonsymmetric model, this simple relation breaks down and $\tau_{\mathrm{ex}}$ is now given by

$$
\tau_{\mathrm{ex}}=\frac{1}{k_{12}+k_{21}},
$$

where $k_{12}$ and $k_{21}$ represent the transition rates between state 1 and state 2 and between state 2 and state 1 , respectively.

Ediger and co-workers ${ }^{2,3}$ speculated that the translational diffusion of a probe molecule between regions with different dynamics could be one of the possible mechanisms of the very slow exchange process observed in experiments. However, such a translational diffusion mediated exchange will scale with the viscosity of the inhomogeneous region. So, $\tau_{\mathrm{ex}}$ will vary similarly as the average equilibrium rotational correlation time, $\langle\tau\rangle_{\text {eq }}$. This does not allow the emergence of the extremely slow relaxation process very near to the glass transition point. ${ }^{2,3}$

Thus the above analysis shows that there must be some other mechanism which determines this very slow relaxation. In fact, the recent Monte Carlo simulation studies of Dasgupta and Valls ${ }^{43}$ for a dense hard sphere system show that the slow relaxation near the glass transition can arise from activated transitions over free energy barriers between different local minima of the free energy. The time scale associated with this transition was found to increase with the average density and this growth of the relaxation time is attributed to an increase of the characteristic height of these free energy barriers. The increase in the transition time rises sharply near the glass transition temperature. This is still just a plausibility.

\section{CONCLUSION}

In this article, the standard form of density functional theory (DFT) has been used to calculate the free energy penalty to create soft localized density fluctuations in a hard sphere liquid. The scaled density has been varied here continuously from 0.99 to 1.09 where we use the uniform liquid of density 1.04 as a reference system.

It is found that the free energy required is much less to create a local inhomogeneity of small size compared to that for a large size. This is attributed to the sharp maximum of the static structure factor $S(k)$ at intermediate wave numbers ( $k \sigma \simeq 2 \pi)$ and also to the very low compressibility of supercooled liquid at low wave numbers. It is shown here that the liquid almost behaves like a homogeneous liquid in the length scale larger than about $5.0 \sigma$ (where $\sigma$ is the molecular diameter) which agrees qualitatively with the recent experimental results. ${ }^{8,9}$ In addition, it is shown here that the inclusion of the surface energy effect will more likely reduce the probability of small size "density droplets" than the droplets of large size. However, it is suggested that the surface effect has a very small contribution to the total free energy cost for forming these droplets.

The results obtained for hard sphere liquid using the Percus-Yevick (PY) approximation for the direct pair correlation function ${ }^{36}$ have been compared with the soft mean spherical approximation $^{37}$ (SMSA) applied to the dense Lennard-Jones liquid. The fluctuations are found to be somewhat larger in size for the continuous potentials due to the increase in spatial correlation length.

Theoretically obtained inhomogeneous probability distributions have been used to calculate both the equilibrium and nonequilibrium orientational correlation functions. We find that the spatially heterogeneous distribution of the density is responsible for the nonexponential nature of the rotational relaxation. For nonequilibrium distribution the average rotational correlation time of molecules relative to that of equilibrium distribution is found to increase with the average density of the liquid. Our theoretical results have been compared with the experimental results of Ediger and co-workers ${ }^{2,3}$ obtained recently by photobleaching technique. Good qualitative agreement is found between the theoretical results presented here and the experimental results.

Relaxation of the nonequilibrium distribution has been studied in a qualitative way using the simple noninteracting two-state exchange model where only two different domains with different densities were considered. The results obtained in this model show that the extremely slow relaxation process observed near the glass transition point ${ }^{2,3}$ cannot be explained by the translational diffusion of a molecule between regions of different dynamics. It is suggested that the transitions between different local minima of the free energy near 
the glass transition could be responsible for this very slow relaxation.

Although we have used the simple two-state model to study the relaxation behavior near the glass transition, an ideal way to consider an inhomogeneous liquid is to consider a probability distribution of the density as given by Kawasaki. ${ }^{44}$ He proposed a probability distribution functional $P(\{\rho\}, t)$ for slow dynamics of the density variable $\rho(\mathbf{r})$ in the supercooled liquid. This probability distribution in the density will lead to a probability distribution of the dynamical variables.

\section{ACKNOWLEDGMENTS}

The authors thank Professor Mark Ediger for many interesting and stimulating discussions and suggestions. Dr. Sarika Bhattacharyya is acknowledged for many helpful discussions and for her help in preparing the manuscript. This work was supported in part by grants from the Council of Scientific and Industrial Research (CSIR), India and the Department of Science and Technology (DST), India. One of the authors (R.M.) thanks the University Grants Commission (UGC) for providing the Research Scholarship.

\footnotetext{
${ }^{1}$ M. D. Ediger, C. A. Angell, and S. R. Nagel, J. Phys. Chem. 100, 13200 (1996).

${ }^{2}$ M. T. Cicerone and M. D. Ediger, J. Chem. Phys. 103, 5684 (1995); F. R. Blackburn, C.-Y. Wang, and M. D. Ediger, J. Phys. Chem. 100, 18249 (1996).

${ }^{3}$ C.-Y. Wang and M. D. Ediger, J. Phys. Chem. B 103, 4177 (1999); C.-Y. Wang and M. D. Ediger, J. Chem. Phys. 112, 6933 (2000).

${ }^{4}$ M. D. Ediger, Annu. Rev. Phys. Chem. 51, 99 (2000).

${ }^{5}$ R. Richert, J. Phys. Chem. B 101, 6323 (1997); R. Richert and M. Richert, Phys. Rev. E 58, 779 (1998); H. Wendt and R. Richert, ibid. 61, 1722 (2000).

${ }^{6}$ K. Schmidt-Rohr and H. Spiess, Phys. Rev. Lett. 66, 3020 (1991); A. Heuer, M. Wilhelm, H. Zimmermann, and H. W. Spiess, ibid. 75, 2851 (1995).

${ }^{7}$ F. Fujara, B. Geil, H. Sillescu, and G. Fleischer, Z. Phys. B: Condens. Matter 88, 195 (1992); I. Chang, F. Fujara, B. Geil, G. Heuberger, T. Mangel, and H. Sillescu, J. Non-Cryst. Solids 172-174, 248 (1994); R. Bohmer, G. Diezemann, B. Geil, and H. Sillescu, Europhys. Lett. 36, 55 (1996); H. Sillescu, J. Non-Cryst. Solids 243, 81 (1999).

${ }^{8}$ M. T. Cicerone, F. R. Blackburn, and M. D. Ediger, J. Chem. Phys. 102, 471 (1995).

${ }^{9}$ U. Tracht, M. Wilhelm, A. Heuer, H. Feng, K. Schmidt-Rohr, and H. W. Spiess, Phys. Rev. Lett. 81, 2727 (1998); U. Tracht, M. Wilhelm, A. Heuer, and H. W. Spiess, J. Magn. Reson. 140, 460 (1999).

${ }^{10}$ D. W. Oxtoby, J. Phys.: Condens. Matter 4, 7627 (1992); D. W. Oxtoby, Acc. Chem. Res. 31, 91 (1998); R. Evans, in Fundamentals of Inhomogeneous Fluids, edited by D. Henderson (Dekker, New York, 1992).

${ }^{11}$ M. D. Ediger, J. Non-Cryst. Solids 235-237, 10 (1998).

${ }^{12}$ G. Adam and J. H. Gibbs, J. Chem. Phys. 43, 139 (1965).

${ }^{13}$ W. Kob, C. Donati, S. J. Plimpton, P. H. Poole, and S. C. Glotzer, Phys.
} Rev. Lett. 79, 2827 (1997).
${ }^{14}$ C. Donati, J. F. Douglas, W. Kob, S. J. Plimpton, P. H. Poole, and S. C. Glotzer, Phys. Rev. Lett. 80, 2338 (1998).

${ }^{15}$ C. Donati, S. C. Glotzer, P. H. Poole, W. Kob, and S. J. Plimpton, Phys. Rev. E 60, 3107 (1999).

${ }^{16}$ R. Yamamoto and A. Onuki, Phys. Rev. E 58, 3515 (1998).

${ }^{17}$ B. Doliwa and A. Heuer, Phys. Rev. Lett. 80, 4915 (1998); J. Phys.: Condens. Matter 11, A277 (1999).

${ }^{18}$ E. R. Weeks, J. C. Crocker, A. C. Levitt, A. Schofield, and D. A. Weitz, Science 287, 627 (2000).

${ }^{19}$ C. T. Moynihan and J. Schroeder, J. Non-Cryst. Solids 160, 52 (1993).

${ }^{20}$ P. Allegrini, J. F. Douglas, and S. C. Glotzer, Phys. Rev. E 60, 5714 (1999).

${ }^{21}$ C. Dasgupta and O. T. Valls, Phys. Rev. E 50, 3916 (1994); O. T. Valls and C. Dasgupta, Transp. Theory Stat. Phys. 24, 1199 (1995).

${ }^{22}$ T. V. Ramakrishnan and M. Yussouff, Phys. Rev. B 19, 2775 (1979).

${ }^{23}$ R. Zwanzig, Chem. Phys. Lett. 164, 639 (1989); J. Chem. Phys. 79, 4507 (1983).

${ }^{24}$ H. T. Davis, J. Am. Ceram. Soc. 60, 499 (1977).

${ }^{25}$ I. Chang and H. Sillescu, J. Phys. Chem. B 101, 8794 (1997).

${ }^{26}$ J. C. Phillips, Phys. Rev. B 32, 5356 (1985); U. Buchenau, M. Prager, N. Nucker, A. J. Dianoux, N. Ahmad, and W. A. Phillipss, ibid. 34, 5665 (1986); M. R. Vukcevich, J. Non-Cryst. Solids 11, 25 (1972); R. E. Youngman, J. Kieffer, J. D. Bass, and L. Duffrene, ibid. 222, 190 (1997).

${ }^{27}$ P. B. Macedo, W. Capps, and T. A. Litovitz, J. Chem. Phys. 44, 3357 (1966).

${ }^{28}$ C. A. Angell, J. Phys. Chem. 75, 3698 (1971); C. A. Angell and K. J. Rao, J. Chem. Phys. 57, 470 (1972).

${ }^{29}$ J. Perez, J. Phys. 46, 427 (1985).

${ }^{30}$ C. H. Wang and E. W. Fischer, J. Chem. Phys. 105, 7316 (1996).

${ }^{31}$ J. Kieffer, J. Phys. Chem. 103, 4153 (1999); J. Kieffer, J. E. Masnik, O. Nickolayev, and J. D. Bass, Phys. Rev. B 58, 694 (1998).

${ }^{32}$ S. Sastry, P. G. Debenedetti, and F. H. Stillinger, Nature (London) 393, 554 (1998).

${ }^{33}$ C. Kaur and S. P. Das, Phys. Rev. Lett. 86, 2062 (2001).

${ }^{34}$ Y. Singh, J. P. Stoessel, and P. G. Wolynes, Phys. Rev. Lett. 54, 1059 (1985)

${ }^{35}$ A. D. J. Haymet and D. W. Oxtoby, J. Chem. Phys. 74, 2559 (1981); D. W. Oxtoby, Adv. Chem. Phys. 70, 263 (1988); D. W. Oxtoby, in Liquids, Freezing and the Glass Transition, Lecture Notes of the Les Houches Summer School, 1989, edited by J. P. Hansen, D. Levesque, and J. ZinnJustin (Elsevier, New York, 1990).

${ }^{36}$ J. P. Hansen and I. R. Mcdonald, Theory of Simple Liquids (Academic, New York, 1986).

${ }^{37}$ W. G. Madden and S. A. Rice, J. Chem. Phys. 72, 4208 (1980).

${ }^{38}$ L. Verlet and J. Weis, Phys. Rev. A 5, 939 (1972).

${ }^{39}$ J. D. Weeks, D. Chandler, and H. C. Andersen, J. Chem. Phys. 54, 5237 (1971).

${ }^{40}$ T. R. Kirkpatrick and P. G. Wolynes, Phys. Rev. B 36, 8552 (1987); T. R. Kirkpatrick, D. Thirumalai, and P. G. Wolynes, Phys. Rev. A 40, 1045 (1989); P. G. Wolynes, in Proceedings of the International Symposium on Frontiers in Science, edited by Shirley S. Chan and Peter G. Debrunner (AIP, New York, 1989), pp. 39-65.

${ }^{41}$ X. Xia and P. G. Wolynes, Proc. Natl. Acad. Sci. U.S.A. 97, 2990 (2000); Phys. Rev. Lett. 86, 5526 (2001).

${ }^{42}$ P. G. Debenedetti, Metastable Liquids (Princeton University Press, Princeton, NJ, 1996).

${ }^{43}$ C. Dasgupta and O. T. Valls, Phys. Rev. E 53, 2603 (1996).

${ }^{44}$ K. Kawasaki, Physica A 208, 35 (1994). 